\title{
Mixing Layer Ignition of Hydrogen
}

\author{
C. TREVIÑO*
}

and

\section{A. LIÑ́íN}

E.T.S.I. Aeronáuticos, U.P.M.,

\begin{abstract}
A theoretical analysis is given for the high-temperature ignition in a laminar mixing layer between hydrogen and air at the high temperatures characteristic of supersonic combustion. We analyze the most important practical case where the temperature of the air stream is higher than that of the hydrogen stream. In this case, the chemical reactions responsible for ignition occur in the air side of the mixing layer, where the mixture is lean. A simplified reduced mechanism is found to describe the ignition process. The radicals $\mathrm{OH}$ and $\mathrm{H}$ follow the steady-state approximation while the radical $\mathrm{O}$ is the chain branching species following an autocatalytic reaction with moderately large activation energy. Numerical results of the governing equations for large values of the activation energy are presented and from a symplified analysis, we obtain a closed form solution of the ignition distance as a function of the physicochemical parameters.
\end{abstract}

\section{INTRODUCTION}

There is increasing interest in the study of ignition and combustion of hydrogen mixtures in a supersonic flow [1]. Here, a high-velocity hot air stream, after crossing an oblique shock wave, is mixed with molecular hydrogen at relatively low temperature. The ignition process then takes place downstream in the mixing layer. Due to the fact that the temperature is much higher in the air stream, the ignition is to be reached in regions close to the air side of this mixing layer. It is necessary to develop simplified models for ignition in mixing layers and this is the main objective of the present work.

The detailed reaction mechanism for hydrogen and oxygen mixtures is very well known, representing the simplest realistic combustion process [2-4]. The understanding of the combustion of the hydrogen/oxygen mixtures is also very important in the hydrocarbon combustion, due to the key role in the radical production zone $[5,6]$. Several studies have been published regarding the ignition of $\mathrm{H}_{2} / \mathrm{O}_{2}$ mixtures [7-11], among others. Recently, efforts have been conducted to deduce a reduced kinetic mechanism that globally contains the essential features of the studied pro- cess [12]. This procedure has been applied to the asymptotic study of premixed and diffusion flames, mainly methane-air [5, 6, 13]. Treviño [14] studied the zeroth-dimensional ignition of stoichiometric $\mathrm{H}_{2} / \mathrm{O}_{2}$ mixtures in an isochoric adiabatic reactor. A reduced kinetic mechanism has been obtained after solving the evolution equations. Three different regimes were identified for the ignition process, depending on the initial temperature and pressure. For initial temperatures larger than the critical temperature (temperature that makes reaction rates of the chain-branching reaction $\mathrm{H}+\mathrm{O}_{2}$ $\rightarrow \mathrm{OH}+\mathrm{O}$ and the chain terminating reaction $\mathrm{H}+\mathrm{O}_{2}+\mathrm{M} \rightarrow \mathrm{HO}_{2}+\mathrm{M}$ equal in magnitude), the ignition can be described as a typical chain branching explosion with no heat release in a first approximation. The reduced kinetic mechanism reduces in this case to only one chain-branching step given by $3 \mathrm{H}_{2}+\mathrm{O}_{2}$ $\rightarrow 2 \mathrm{H}_{2} \mathrm{O}+2 \mathrm{H}$, with the rate of the elementary reaction $\mathrm{H}+\mathrm{O}_{2} \rightarrow \mathrm{OH}+\mathrm{O}$. In the high-temperature ignition process, more fuel is consumed as compared with the stoichiometry, making this process to be very rich. However, for lean and extremely lean mixtures, the fuel concentration is very low and then the fuel consumption reactions are not fast enough to 
make both $\mathrm{O}$ and $\mathrm{OH}$ to be in steady state. In this case $H$ reaches the quasi-steady-state behavior, because the chain-branching reaction $\mathrm{H}+\mathrm{O}_{2} \rightarrow \mathrm{OH}+\mathrm{O}$ is very fast compared with the other important shuffle reactions. It is necessary to deduce another global mechanism appropriate to lean mixtures. As the temperature decreases to values close to the critical temperature, the heat release has to be retained in the analysis to improve the deduced ignition time. As the temperature decreases further, the chain-branching reaction loses in importance and the evolution of intermediate species controls the process. A thermal runaway is produced in this regime characterizing the ignition process at low initial temperatures. In all three regimes, an analytical expression has been obtained for the ignition delay time. The reduced kinetic mechanism obtained systematically from the homogeneous reactor analysis, can be applied to nonhomogeneous mixtures, like in the mixing layer studied here. In this case, assuming that the temperature in both sides of the mixing layer is higher than the crossover temperature, there are two appropriate reduced kinetic mechanisms for lean and rich mixtures. However, for a big temperature difference in both streams, only one is the important mechanism because the kinetics on the other side is too slow to be considered and can be assumed to be frozen.

The ignition problem of a combustible gas by a hot flat plate in a boundary layer flow has been studied using an irreversible, one-step global chemical reaction based on large activation energy [15-17]. In the last of the works, a three-term asymptotic series has been obtained for the ignition Damköhler number. The correction to the leading-order Zeldovich approximation can be very important for moderate values of the Zeldovich numbers. Using a two-step reaction mechanism based in the Zeldovich mechanism [18], Niioka [19] studied the chain-branching ignition process of a cold combustible gas by hot inert gas. Treviño and Méndez [20] employed the global reduced kinetic mechanisms for the high- and lowtemperature regimes obtained in Ref. 14 to study the ignition process of hydrogen mixtures by a hot flat plate in a boundary layer flow. They obtained a closed form solution for the ignition distance as a function of the physicochemical parameters.

The main objective of the present study is to analyze the practical case of the ignition of hydrogen and hot air in a laminar mixing layer. An appropriate reduced kinetic mechanism valid for lean mixtures is to be obtained and

TABLE 1

Mechanism of the Hydrogen Oxygen Mixtures

\begin{tabular}{|c|c|c|c|}
\hline$k=A T^{n} \exp (-E / R T)$ & $A(\mathrm{~cm}, \mathrm{~mol}, \mathrm{~s})$ & $n$ & $E(K J / \mathrm{mol})$ \\
\hline$(1,2) \mathrm{H}+\mathrm{O}_{2} \Leftrightarrow \mathrm{OH}+\mathrm{O}$ & $2.0010^{14}$ & 0.0 & 70.30 \\
\hline$(3,4) \mathrm{H}_{2}+\mathrm{O} \Leftrightarrow \mathrm{OH}+\mathrm{H}$ & $5.0610^{04}$ & 2.7 & 26.30 \\
\hline$(5,6) \mathrm{H}_{2}+\mathrm{OH} \Leftrightarrow \mathrm{H}_{2} \mathrm{O}+\mathrm{H}$ & $1.0010^{08}$ & 1.6 & 13.80 \\
\hline$(7,8) \mathrm{OH}+\mathrm{OH} \Leftrightarrow \mathrm{H}_{2} \mathrm{O}+\mathrm{O}$ & $1.5010^{09}$ & 1.1 & 0.40 \\
\hline$(9,10) \mathrm{H}+\mathrm{O}_{2}+\mathrm{M} \Leftrightarrow \mathrm{HO}_{2}+\mathrm{M}$ & $2.3010^{18}$ & -0.8 & 0.00 \\
\hline$(11,12) \mathrm{HO}_{2}+\mathrm{H} \Leftrightarrow \mathrm{OH}+\mathrm{OH}$ & $1.5010^{14}$ & 0.0 & 4.20 \\
\hline$(13,14) \mathrm{HO}_{2}+\mathrm{H} \Leftrightarrow \mathrm{H}_{2}+\mathrm{O}_{2}$ & $2.5010^{13}$ & 0.0 & 2.90 \\
\hline$(15,16) \mathrm{HO}_{2}+\mathrm{OH} \Leftrightarrow \mathrm{H}_{2} \mathrm{O}+\mathrm{O}_{2}$ & $6.0010^{13}$ & 0.0 & 0.00 \\
\hline$(17,18) \mathrm{HO}_{2}+\mathrm{H} \Leftrightarrow \mathrm{H}_{2} \mathrm{O}+\mathrm{O}$ & $3.0010^{11}$ & 0.0 & 7.20 \\
\hline$(19,20) \mathrm{HO}_{2}+\mathrm{O} \Leftrightarrow \mathrm{OH}+\mathrm{O}_{2}$ & $1.8010^{11}$ & 0.0 & -1.70 \\
\hline$(21,22) \mathrm{HO}_{2}+\mathrm{HO}_{2} \Leftrightarrow \mathrm{H}_{2} \mathrm{O}_{2}+\mathrm{O}_{2}$ & $2.5010^{11}$ & 0.0 & -5.20 \\
\hline$(23,24) \mathrm{OH}+\mathrm{OH}+\mathrm{M} \Leftrightarrow \mathrm{H}_{2} \mathrm{O}_{2}+\mathrm{M}$ & $3.2510^{22}$ & -2.0 & 0.00 \\
\hline$(25,26) \mathrm{H}_{2} \mathrm{O}_{2}+\mathrm{H} \Leftrightarrow \mathrm{H}_{2}+\mathrm{HO}_{2}$ & $1.7010^{10}$ & 0.0 & 15.70 \\
\hline$(27,28) \mathrm{H}_{2} \mathrm{O}_{2}+\mathrm{H} \Leftrightarrow \mathrm{H}_{2} \mathrm{O}+\mathrm{OH}$ & $1.0010^{11}$ & 0.0 & 15.00 \\
\hline$(29,30) \mathrm{H}_{2} \mathrm{O}_{2}+\mathrm{O} \Leftrightarrow \mathrm{OH}+\mathrm{HO}_{2}$ & $2.8010^{11}$ & 0.0 & 26.80 \\
\hline$(31,32) \mathrm{H}_{2} \mathrm{O}_{2}+\mathrm{OH} \Leftrightarrow \mathrm{H}_{2} \mathrm{O}+\mathrm{HO}_{2}$ & $5.4010^{10}$ & 0.0 & 4.20 \\
\hline$(33,34) \mathrm{H}_{2}+\mathrm{M} \Leftrightarrow \mathrm{H}+\mathrm{H}+\mathrm{M}$ & $6.7610^{16}$ & -1.0 & 436.26 \\
\hline$(35,36) \mathrm{H}+\mathrm{OH}+\mathrm{M} \Leftrightarrow \mathrm{H}_{2} \mathrm{O}+\mathrm{M}$ & $2.2010^{20}$ & -2.0 & 0.00 \\
\hline$(37,38) \mathrm{O}+\mathrm{O}+\mathrm{M} \Leftrightarrow \mathrm{O}_{2}+\mathrm{M}$ & $2.9010^{15}$ & -1.0 & 0.00 \\
\hline
\end{tabular}


applied to the ignition process. A simplified closed form solution for the ignition distance in a mixing layer is to be deduced.

\section{REDUCED KINETIC MECHANISM FOR THE HIGH-TEMPERATURE LEAN REGIME}

The ignition process of hydrogen with oxygen changes dramatically, depending on the initial gas temperature and the equivalence ratio of the mixture. In a nonhomogeneous system, there are different reduced mechanisms appropriate for different zones, depending on temperature and species concentrations. We assume in this work that the air side temperature is much larger than the fuel side temperature and also is much larger than a critical temperature obtained from the competition between the chain branching reaction 1 and the chain terminating reaction 9 , listed in Table 1 . This crossover temperature is denoted by $T_{c}$, which is around $1000 \mathrm{~K}$ for $1 \mathrm{~atm}$ pressure. There are two reduced kinetic mechanisms appropriate for this case, one valid for lean mixtures close to the air side and one valid for stoichiometric to rich mixtures. From a study of the ignition process in a homogeneous isochoric reactor, Treviño and Liñán [21], found that the following elementary reactions are important for lean mixtures at high initial temperatures (far away from $T_{c}$ ) and the reduced kinetic mechanism obtained is the relevant one to be applied at the air side of the mixing layer, where the temperature is higher. The another mechanism valid for rich and stoichiometric mixtures is not considered in this work because the reactions in this zone are very slow compared with the reactions on the air side. At the beginning (no radicals), reaction 14 ,

$$
\mathrm{H}_{2}+\mathrm{O}_{2} \Leftrightarrow \mathrm{HO}_{2}+\mathrm{H}
$$

is the important initiation step. Once trace amounts of radicals are present, this reaction has no more influence on the process in this high temperature limit. However, in the case of hot air stream, atomic oxygen radicals are present due to air dissociation, which makes easier to ignite the mixture, thus decreasing the ignition distance. From this point up to the end, the three forward shuffle reactions are very important and have to be retained in the analysis of the ignition process. These reactions are

$$
\begin{aligned}
& \mathrm{H}+\mathrm{O}_{2} \Rightarrow \mathrm{OH}+\mathrm{O} \\
& \mathrm{H}_{2}+\mathrm{O} \Rightarrow \mathrm{OH}+\mathrm{H} \\
& \mathrm{H}_{2}+\mathrm{OH} \Rightarrow \mathrm{H}_{2} \mathrm{O}+\mathrm{H}
\end{aligned}
$$

Because $\mathrm{H}_{2}$ is consumed rapidly in the ignition process of lean mixtures, reaction 7 becomes the most important $\mathrm{OH}$ consumer reaction,

$$
\mathrm{OH}+\mathrm{OH} \Rightarrow \mathrm{H}_{2} \mathrm{O}+\mathrm{O} \text {. }
$$

$\mathrm{HO}_{2}$ is produced mainly through reaction 9 , whose rate is very slow compared with the other reactions for high initial temperatures,

$$
\mathrm{H}+\mathrm{O}_{2}+\mathrm{M} \Rightarrow \mathrm{HO}_{2}+\mathrm{M}
$$

$\mathrm{HO}_{2}$ is being consumed mainly by reactions 15 and 19

$$
\begin{aligned}
& \mathrm{HO}_{2}+\mathrm{OH} \Rightarrow \mathrm{H}_{2} \mathrm{O}+\mathrm{O}_{2}, \\
& \mathrm{HO}_{2}+\mathrm{O} \Rightarrow \mathrm{OH}+\mathrm{O}_{2} .
\end{aligned}
$$

For very lean mixtures, reactions 3 and 5 are not very fast due to the low concentration of molecular hydrogen and become the rate limiting reactions in the ignition process. Reaction 1 now is fast compared with 3 and 5 and makes the hydrogen atom to behave in quasi-steadystate. The backward reactions (mainly radicalradical) have no influence on the ignition process. It was found that the radicals $\mathrm{OH}, \mathrm{H}$, and $\mathrm{HO}_{2}$ are produced and consumed at rates much larger than the radical $O$. Therefore, the species $\mathrm{H}, \mathrm{OH}$, and $\mathrm{HO}_{2}$ can be assumed to be in steady-state. The reduced kinetic mechanism then reduces to

$$
\begin{aligned}
& \text { I } \mathrm{H}_{2}+\mathrm{O}_{2} \stackrel{3+5+14}{\Rightarrow} \mathrm{H}_{2} \mathrm{O}+\mathrm{O}, \\
& \text { II } \mathrm{O}+\mathrm{O} \stackrel{9}{\Rightarrow} \mathrm{O}_{2},
\end{aligned}
$$

where the number above the arrow represents the rate of the elementary reaction associated with the global step. From the steady-state assumptions for $\mathrm{H}$ and $\mathrm{OH}$, the concentrations of these radicals are related to that of atomic 
oxygen as

$$
\begin{aligned}
\frac{C_{\mathrm{H}}}{C_{\mathrm{H}_{2}}}= & \frac{k_{14}}{k_{1}+\bar{k}_{9}}+\frac{k_{3} C_{\mathrm{O}}}{\left(k_{1}+\bar{k}_{9}\right) C_{\mathrm{O}_{2}}} \\
& +\frac{k_{5} \sqrt{k_{3} C_{\mathrm{H}_{2}} C_{\mathrm{O}}}}{\left(k_{1}+\bar{k}_{9}\right) \sqrt{k_{7}} C_{\mathrm{O}_{2}}}, \\
C_{\mathrm{OH}}= & \sqrt{\frac{k_{3} C_{\mathrm{H}_{2}} C_{\mathrm{O}}}{k_{7}}},
\end{aligned}
$$

where $C_{i}$ corresponds to the molar concentration of species $i$ and $k_{j}$ is the reaction rate of reaction $j . \bar{k}_{9}$ corresponds to the equivalent two-body reaction rate of the three body reaction 9. The reaction rates of the two global steps are then given by

$$
\begin{gathered}
w_{I}=k_{14} C_{\mathrm{O}_{2}} C_{\mathrm{H}_{2}}+k_{3} C_{\mathrm{O}} C_{\mathrm{H}_{2}} \\
+\frac{k_{5} \sqrt{k_{3} C_{\mathrm{H}_{2}}^{3} C_{\mathrm{O}}}}{\sqrt{k_{7}}}, \\
w_{\mathrm{II}}=\frac{\bar{k}_{9} w_{\mathrm{I}}}{k_{1}+\bar{k}_{9}} .
\end{gathered}
$$

For high initial temperatures, the reaction rate of reaction 9 is very small compared to reaction 1 , that is $k_{1} \gg \bar{k}_{9}$ and then the second global step is very slow and can be neglected in a first approximation for this ignition process. Global step I corresponds to a chain branching step with radical $O$ as the chain branching species. Figure 1 shows the ignition delay time for stoichiometric and lean hydrogen/air mixtures in an homogeneous isochoric reactor, computed numerically for an initial temperature of $2000 \mathrm{~K}$ with full kinetics (solid line) as a function of the equivalence ratio. The asymptotic solution obtained using the above reduced kinetic mechanism is also plotted (dotted line) in this figure, giving very good agreement with the numerical results and following the same trend with the equivalence ratio. The asymptotic relationship obtained using the overall global step

$3 \mathrm{H}_{2}+\mathrm{O}_{2} \Rightarrow 2 \mathrm{H}_{2} \mathrm{O}+2 \mathrm{H}$

obtained for stoichiometric and rich mixtures is also plotted. This relationship shows the inverse behavior of the ignition delay time with

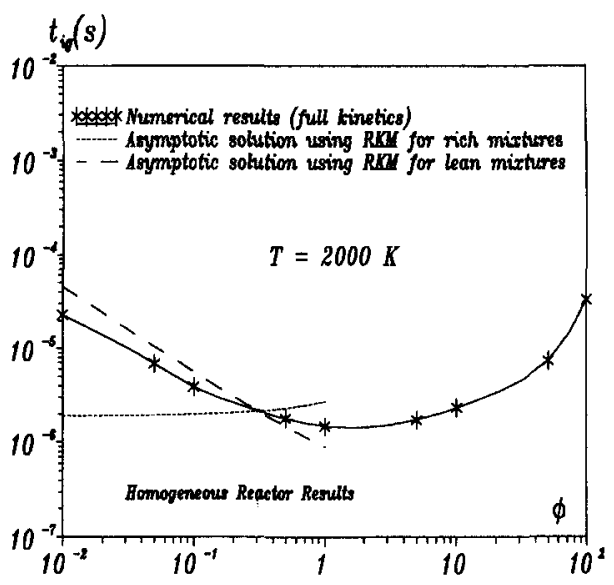

Fig. 1. Numerical and asymptotic solution for the ignition delay time for an homogeneous reactor as a function of the equivalence ratio for an initial temperature of $T_{0}=$ $2000 \mathrm{~K}$.

the equivalence ratio. Therefore, the appropriate reduced kinetic mechanism corresponds to global steps I and II. The production rate of atomic oxygen radical has three sources given by elementary reactions 14,3 , and 5 . Reaction 14 is extremely slow and could be neglected if we assume that radicals are present in the hot air due to dissociation. Reaction 5 is very slow compared with reaction 3 because both $\mathrm{H}_{2}$ and $\mathrm{OH}$ concentrations are very low in lean mixtures. Reaction 3 is the most important reaction in this case. In the next section we develop an asymptotic analysis by neglecting the contribution of reaction 5 in the ignition process.

\section{FORMULATION}

Assuming the global mechanism for the high temperature ignition of lean hydrogen mixtures to be given by reaction I (with very slow reaction II), the nondimensional isovelocity mixing layer governing equations of a flow of hydrogen co-flowing with hot air, are given by

$$
\begin{aligned}
\chi \frac{\partial Y_{\mathrm{H}_{2}}}{\partial \chi} & -\frac{\eta}{2} \frac{\partial Y_{\mathrm{H}_{2}}}{\partial \eta}-\frac{1}{L} \frac{\partial^{2} Y_{\mathrm{H}_{2}}}{\partial \eta^{2}} \\
= & -\frac{1}{8} \chi Y_{\mathrm{H}_{2}} Y_{\mathrm{O}} \exp \left(\frac{\beta(\theta-1)}{\theta}\right)
\end{aligned}
$$




$$
-\frac{f_{14}}{16} \chi Y_{\mathrm{H}_{2}} Y_{\mathrm{O}_{2}} \exp \left(\frac{\beta_{14}(\theta-1)}{\theta}\right),
$$

$$
\begin{aligned}
\chi \frac{\partial Y_{\mathrm{O}}}{\partial \chi} & -\frac{\eta}{2} \frac{\partial Y_{\mathrm{O}}}{\partial \eta}-\frac{\partial^{2} Y_{\mathrm{O}}}{\partial \eta^{2}} \\
= & \chi Y_{\mathrm{H}_{2}} Y_{\mathrm{O}} \exp \left(\frac{\beta(\theta-1)}{\theta}\right) \\
& +\frac{f_{14}}{2} \chi Y_{\mathrm{H}_{2}} Y_{\mathrm{O}_{2}} \exp \left(\frac{\beta_{14}(\theta-1)}{\theta}\right),
\end{aligned}
$$

$$
\begin{aligned}
\chi \frac{\partial Y_{\mathrm{O}_{2}}}{\partial \chi} & -\frac{\eta}{2} \frac{\partial Y_{\mathrm{O}_{2}}}{\partial \eta}-\frac{\partial^{2} Y_{\mathrm{O}_{2}}}{\partial \eta^{2}} \\
= & -\frac{2}{y_{\mathrm{O}_{2 x}}} \chi Y_{\mathrm{H}_{2}} Y_{\mathrm{O}} \exp \left(\frac{\beta(\theta-1)}{\theta}\right) \\
& -\frac{f_{14}}{y_{\mathrm{O}_{2 x}}} \chi Y_{\mathrm{H}_{2}} Y_{\mathrm{O}_{2}} \exp \left(\frac{\beta_{14}(\theta-1)}{\theta}\right),
\end{aligned}
$$

$$
-\frac{\eta}{2} \frac{\partial \theta}{\partial \eta}-\frac{\partial^{2} \theta}{\partial \eta^{2}}=0
$$

where the nondimensional variables are defined as

$$
\theta=\frac{T}{T_{\infty}}, \quad \chi=\frac{x}{U_{\infty} t_{\infty}}, \quad \eta=y \sqrt{\frac{U_{\infty}}{\alpha x}},
$$$$
Y_{\mathrm{O}}=y_{\mathrm{O}}, \quad Y_{\mathrm{H}_{2}}=\frac{y_{\mathrm{H}_{2}}}{y_{\mathrm{H}_{2 x}}}, \quad Y_{\mathrm{O}_{2}}=\frac{y_{\mathrm{O}_{2}}}{y_{\mathrm{O}_{2 x}}}
$$$$
\beta=\frac{\bar{E}_{3}}{R T_{\infty}}, \quad \beta_{14}=\frac{E_{14}}{R T_{\infty}},
$$

$f_{14}=\frac{A_{14} y_{\mathrm{O}_{2 x}}}{\overline{A_{3}}} \exp \left(\beta-\beta_{14}\right)$,

$t_{\infty}=\frac{W_{\mathrm{H}_{2}} \exp (\beta)}{\overline{A_{3}} \rho_{\infty} y_{\mathrm{H}_{2 x}}}$

The boundary conditions are given by

$\eta \rightarrow \infty ; \quad Y_{\mathrm{H}_{2}}=0, \quad \theta=Y_{\mathrm{O}_{2}}=1$,

$Y_{\mathrm{O}}=Y_{\mathrm{O} x}$

$\eta \rightarrow-\infty ; \quad Y_{\mathrm{O}}=Y_{\mathrm{O}_{2}}=0, \quad Y_{\mathrm{H}_{2}}=1$,

$\theta=1-\theta_{d}$, with $\theta_{d}=\left(T_{\infty}-T_{-\infty}\right) / T_{\infty}$. In the above equations, $x$ is the longitudinal coordinate parallel to both streams, with the origin at the point where the mixing layer begins. $y$ corresponds to the transversal coordinate. The fuel side is for $y<0$. $\alpha$ corresponds to the thermal diffusivity, $\alpha=\lambda /\left(\rho C_{p}\right)$, where $\lambda$ is the thermal conductivity, $\rho$ is the fluid density and $C_{p}$ is the specific heat at constant pressure. $U_{\infty}$ is the fluid velocity assumed to be equal in both streams. $y_{i}$ corresponds to the mass concentration of the species $i$ in the mixture. $T_{\infty}$ and $T_{-\infty}$ correspond to the air and fuel temperature far away the mixing layer, respectively. $L$ is the Lewis number of the molecular hydrogen. $\overline{A_{3}}$ represents the effective pre-exponential factor for elementary chemical reaction 3 , which in this case is $\bar{A}_{3}=A_{3} T_{\infty}^{2.7} \exp (2.7)$, and $\bar{E}_{3}$ is the corresponding Arrhenius activation energy for reaction 3 given by $\bar{E}_{3}=E_{3}+$ $2.7 R T_{\infty}$. It was assumed that the energy release is negligible in this ignition process because the reaction takes place in a region with a very lean mixture. The evolutive ignition problem also requires a radical concentration at the air side, which can be obtained from the equilibrium conditions in the air stream $\left(\mathrm{O}_{2} \Leftrightarrow 2 \mathrm{O}\right)$ downstream of the oblique shock wave. Assuming reactions 37 and 38 to be in partial equilibrium, the radical concentration at the air side, $Y_{\mathrm{O} \infty}$ can be obtained after solving the quadratic equation

$\frac{k_{37}}{k_{38}} \frac{W_{\mathrm{O}_{2}}}{W_{\mathrm{O}}^{2}} Y_{\mathrm{O} \infty}^{2}+Y_{\mathrm{O} \infty}=y_{\mathrm{O}_{2 \nsim}}$.

Therefore, the ignition can be treated as a pure chain branching process. The solution of the energy Eq. 8, for the frozen temperature distribution is then

$\theta=1-\frac{\theta_{d}}{2} \operatorname{erfc}\left(\frac{\eta}{2}\right)$

where erfc denotes the complementary error function. In the following sections we analyze the ignition process using two different approaches. First we use the quasi-steady approximation in order to obtain the critical value of $\chi$, downstream of which diffusion is not strong enough to stop the chemical reaction. This 
critical value denotes a bifurcation point. In the second approach, we use numerical techniques to solve the evolution problem (Eqs. 4 to 9) and compare the results from both approaches. Finally, a simple formula is to be deduced for the ignition distance.

\section{QUASI-STEADY APPROACH}

Assuming a large activation energy for elementary reaction 3 , the chemical reaction is only important in regions close to the air side, where the temperature is the highest. The size of the inner reactive region is dictated by the decrease of the mixture temperature and corresponds to the region where the temperature decreases in a quantity of the FrankKamenetskii order. For large values of $\eta$, the behavior of the nondimensional temperature, $\theta$, can be written as

$$
\theta \sim 1-\theta_{d} \frac{\exp \left(-\frac{\eta^{2}}{4}\right)}{\sqrt{\pi} \eta}, \text { for } \eta \rightarrow \infty
$$

We can define a position $\eta_{r}$ where the temperature decreases in a quantity of the FrankKamenetskii order, that is $(1-\theta) \beta \sim 1$. In a first approximation, $\eta_{r}$ can be obtained from the relationship

$$
\frac{\exp \left(-\frac{\eta_{r}^{2}}{4}\right)}{\eta_{r}}=\frac{\sqrt{\pi}}{\beta \theta_{d}},
$$

where $\eta_{r}$ is assumed to be a value moderately large compared with unity. The inner reactive zone is then localized in regions where $\eta \sim \eta_{r}$ to $\eta \rightarrow \infty$. In the quasi-steady approach we neglect the derivative in the time-like coordinate $\chi$ in order to obtain a critical distance, $\chi_{c}$, beyond which diffusion is not dominant. Introducing the following inner variables

$z=\frac{Y_{\mathrm{O}}}{8} ; \quad \xi=\exp \left[-\frac{\eta_{r}\left(\eta-\eta_{r}\right)}{2}\right]$,

the conservation equations for the fuel and radical concentrations can be simplified in a first approximation to

$$
\begin{aligned}
& \frac{\xi^{2} \eta_{r}^{2}}{4} \frac{\partial^{2} z}{\partial \xi^{2}}=-\chi Y_{\mathrm{H}_{2}} z \exp (-\xi), \\
& \frac{\xi \eta_{r}^{2}(1-L)}{4 L} \frac{\partial Y_{\mathrm{H}_{2}}}{\partial \xi}+\frac{\xi^{2} \eta_{r}^{2}}{4 L} \frac{\partial^{2} Y_{\mathrm{H}_{2}}}{\partial \xi^{2}} \\
& =\chi Y_{\mathrm{H}_{2}} z \exp (-\xi),
\end{aligned}
$$

where also we neglected the initiation reaction 14. The boundary conditions associated with the inner Eqs. 15 and 16 are the following. Towards the air side $\xi=0$, both radical and fuel concentrations disappear

$z=Y_{\mathrm{H}_{2}}=0$ for $\xi=0$.

At the rand of the inner reactive radical production zone towards the fuel side $(\xi \rightarrow \infty)$, the radical concentration achieves a maximum value and diffuses into the outer nonreactive zone, that is,

$\frac{\partial z}{\partial \xi}=0$ for $\xi \rightarrow \infty$.

The other boundary condition arises from the matching conditions with the nonreactive region $(\xi \rightarrow \infty)$. It requires that the fuel concentration gradient matches with the frozen flow solution of the outer nonreactive zone and is given by

$$
\frac{\partial Y_{\mathrm{H}_{2}}}{\partial \xi} \sim Y_{\mathrm{H}_{2} r f} L \xi^{L-1},
$$

where $\mathrm{Y}_{\mathrm{H}_{2} r f}$ corresponds to the frozen concentration of the fuel at the position $\eta=\eta_{r}$. Introducing an appropriate nondimensional coordinate, $\bar{\chi}$ as

$\bar{\chi}=\frac{4 \chi}{\eta_{r}^{2}}$

Eqs. 15 and 16 then take the form

$$
\begin{gathered}
\frac{\partial^{2} z}{\partial \xi^{2}}=-\frac{\bar{\chi} Y_{\mathrm{H}_{2}} z \exp (-\xi)}{\xi^{2}}, \\
\frac{(1-L)}{\xi L} \frac{\partial Y_{\mathrm{H}_{2}}}{\partial \xi}+\frac{1}{L} \frac{\partial^{2} Y_{\mathrm{H}_{2}}}{\partial \xi^{2}} \\
=\frac{\bar{\chi} Y_{\mathrm{H}_{2}} z \exp (-\xi)}{\xi^{2}} .
\end{gathered}
$$


This system of equations admits two solutions. The first one corresponds to the trivial solution $z=0$ and $Y_{\mathrm{H}_{2}}=Y_{\mathrm{H}_{2} f}$. However, for values of $\bar{\chi}$, larger than a critical one, the solution bifurcates to an autocatalytic ignited branch. This critical value can be obtained after solving the linearized version of Eqs. 17 to 21. Adding Eqs. 20 and 21, integrating once and applying the boundary condition at the air side $(\xi=0)$, we obtain the following coupling relationship

$Y_{\mathrm{H}_{2}}+z=\xi\left[\frac{1}{L} \frac{\partial Y_{\mathrm{H}_{2}}}{\partial \xi}+\frac{\partial z}{\partial \xi}\right]$.

With the aid of the matching condition 19, the boundary condition at the fuel side of the reaction zone $(\xi \rightarrow \infty)$ takes the form

$Y_{\mathrm{H}_{2}}+z_{\infty} \sim Y_{\mathrm{H}_{2} r f} \xi^{L}$,

where $z_{\infty}$ corresponds to the maximum value of the nondimensional radical concentration, $z$. Introducing the following variables, $\sigma(\xi)$ and $\phi(\xi)$, in the form

$Y_{\mathrm{H}_{2}}=Y_{\mathrm{H}_{2} r f} \sigma \xi^{L} ; \quad z=Y_{\mathrm{H}_{2 r f}} \phi$,

the inner reactive zone governing Eqs. 20 and 22 take the form

$\frac{\partial^{2} \phi}{\partial \xi^{2}}=-\frac{\Delta \phi \sigma \exp (-\xi)}{\xi^{2-L}}$,

$\phi-\xi \frac{\partial \phi}{\partial \xi}=\frac{\xi^{L+1}}{L} \frac{\partial \sigma}{\partial \xi}$.

The boundary conditions transform to

$\left.\phi(\xi=0)=0 ; \quad \frac{\partial \phi}{\partial \xi}\right]_{\xi \rightarrow \infty}=0 ;$

$\sigma(\xi \rightarrow \infty) \sim 1-\frac{\phi_{\infty}}{\xi^{L}}$,

where

$\Delta=\bar{\chi} Y_{\mathrm{H}_{2} r f}=\frac{4 \chi Y_{\mathrm{H}_{2} r f}}{\eta_{r}^{2}}$

with

$Y_{\mathrm{H}_{2} r f}=\frac{1}{2} \operatorname{erfc}\left(-\frac{\eta_{r} \sqrt{L}}{2}\right)$.
There are two solutions to the problem given by Eqs. 25-27. The first one corresponds to the trivial (frozen) solution given by $\phi=0$ and $\sigma=1$. However, for values of $\Delta$ larger than a critical value denoted by $\Delta_{c}$, there is a nontrivial ignited branch solution. In order to obtain the critical value of the nondimensional length, $\Delta_{c}$, we assume a very small radical concentration everywhere, that is

$$
\begin{aligned}
& \phi=\epsilon \phi_{0}+\epsilon^{2} \phi_{1}+\cdots \\
& \sigma=1-\epsilon \sigma_{1}+\cdots \\
& \Delta=\Delta_{c}+\epsilon \Delta_{1}+\cdots,
\end{aligned}
$$

where $\epsilon$ is a small number and $\phi_{0}$ is scaled in such a way that $\phi_{0}(\xi \rightarrow \infty)=\phi_{0 \infty}=1$. Introducing the relationships given by Eq. 29 into the species governing equations in the reactive zone, we obtain the following system of equations by collecting terms with the same power of $\epsilon$ :

$$
\begin{aligned}
& \frac{\partial^{2} \phi_{0}}{\partial \xi^{2}}+\frac{\Delta_{c} \phi_{0} \exp (-\xi)}{\xi^{2-L}}=0, \\
& \frac{\partial^{2} \phi_{1}}{\partial \xi^{2}}+\frac{\Delta_{c} \phi_{1} \exp (-\xi)}{\xi^{2-L}} \\
& =\left(\Delta_{c} \sigma_{1}-\Delta_{1}\right) \frac{\phi_{0} \exp (-\xi)}{\xi^{2-L}}, \\
& \frac{\partial \sigma_{1}}{\partial \xi}=\left(\xi \frac{\partial \phi_{0}}{\partial \xi}-\phi_{0}\right) \frac{L}{\xi^{L+1}},
\end{aligned}
$$

etc., with the boundary conditions

$$
\begin{aligned}
\phi_{0}(\xi=0) & \left.=\phi_{1}(\xi=0)=\frac{\partial \phi_{0}}{\partial \xi}\right]_{\xi \rightarrow \infty} \\
& \left.=\frac{\partial \phi_{1}}{\partial \xi}\right]_{\xi \rightarrow \infty}=0
\end{aligned}
$$

$\sigma_{1}(\xi \rightarrow \infty) \sim \frac{1}{\xi^{L}}$

Equation 30 with the corresponding boundary conditions given in Eq. 33 represents an eigenvalue problem and has a nontrivial solution for only one value of the parameter $\Delta_{c}$, that is the critical value for ignition of the autocatalytic reaction. Figure 2 shows this critical value of 


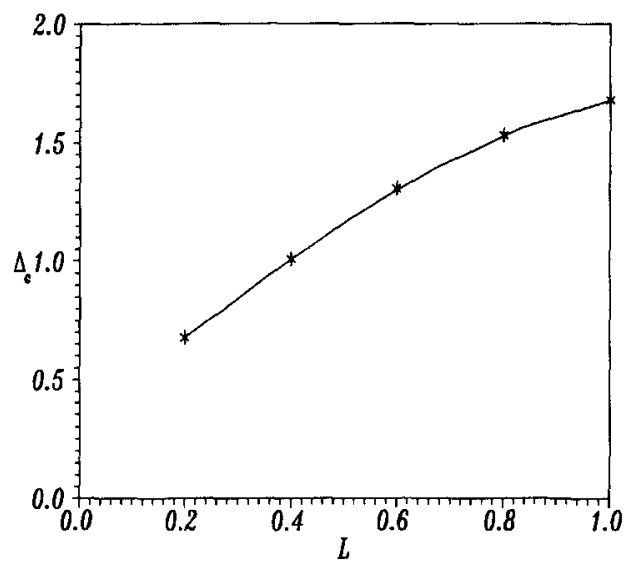

Fig. 2. Critical reduced Damköhler number for ignition of the autocatalytic reaction, as a function of the fuel Lewis number.

the reduced Damköhler number $\Delta_{c}$ as a function of the Lewis number of the fuel, $L$. The solution of the system of Eqs. 31-33 gives the form of the bifurcated ignited branch for small values of the parameter $\epsilon \sim \Delta-\Delta_{c}$. The solution can be obtained by using the solvability condition

$\int_{0}^{\infty}\left(\Delta_{c} \sigma_{1}-\Delta_{1}\right) \frac{\Phi_{0}{ }^{2} \exp (-\xi)}{\xi^{2}-L} d \xi=0$.

For the specific case of the fuel Lewis number of $L=0.2$, we obtained $\Delta_{c}=0.67 \ldots$ and $\Delta_{1}$ $=0.4343 \ldots$ Figure 3 shows the eigenfunctions $\phi_{0}$ and $\sigma_{1}$ as a function of $\xi$. Now $\epsilon$ can be removed from Eqs. 29, giving in a first approxi-

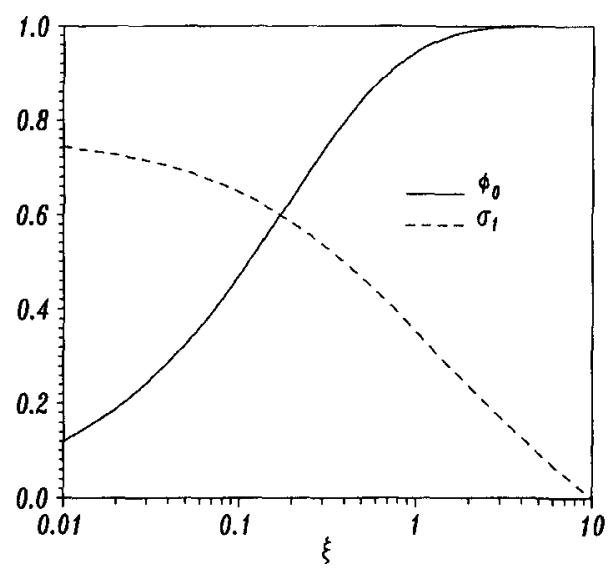

Fig. 3. Eigenfunctions $\phi_{0}$ and $\sigma_{1}$ as a function of the nondimensional longitudinal coordinate, $\xi$. mation the maximum value of the radical concentration as a function of the nondimensional longitudinal coordinate as

$\phi_{\infty} \simeq \frac{\Delta-\Delta_{c}}{\Delta_{1}}$, for $\Delta \rightarrow \Delta_{c}$.

For large values of $\Delta$, there is a reaction zone around $\xi=\xi_{0} \gg 1$, of thickness unity. This zone separates an equilibrium region, $\xi<$ $\xi_{0}$ with no molecular hydrogen, from an inert zone $\xi>\xi_{0}$ where the chemical reaction is frozen. In order to study this case, it is convenient to redefine the independent variable as

$\zeta=\exp \left(-\frac{\eta_{r} L\left(\eta-\eta_{r}\right)}{2}\right)$

instead of $\xi$ introduced in Eq. 14. The fuel and radical concentration Eqs. 20 and 21 reduce to

$\frac{\partial^{2} y_{2}}{\partial \zeta^{2}}=\frac{\Delta y_{2} \phi \exp \left(-\zeta^{\frac{1}{L}}\right)}{\zeta^{2}}$

$\frac{(1-L)}{\zeta} \frac{\partial \phi}{\partial \zeta}-L \frac{\partial^{2} \phi}{\partial \zeta^{2}}=\frac{\Delta y_{2} \phi \exp \left(-\zeta^{\frac{1}{L}}\right)}{\zeta^{2}}$

where

$Y_{\mathrm{H}_{2}}=Y_{\mathrm{H}_{2 r f}} y_{2} ; \quad z=Y_{\mathrm{H}_{2} r f} \phi$

The boundary conditions transform to

$\left.\phi(\zeta=0)=y_{2}(\zeta=0)=\frac{\partial \phi}{\partial \zeta}\right]_{\zeta \rightarrow \infty}=0 ;$

$\left.\frac{\partial y_{2}}{\partial \zeta}\right]_{\zeta \rightarrow \infty}=1$

Adding Eqs. 37 and 38 and integrating once we obtain

$\phi+y_{2}=\zeta\left(\frac{\partial y_{2}}{\partial \zeta}+L \frac{\partial \phi}{\partial \zeta}\right)$ 
Using the matching condition with the inert region, we obtain for large values of $\zeta$

$\phi_{\infty}+y_{2} \sim \zeta$, for $\zeta \rightarrow \infty$.

For large values of $\Delta$, the reaction located around $\zeta_{0}$ separates an equilibrium region, $\zeta<\zeta_{0}$ with $y_{2}=0$ from the inert region, $\zeta>$ $\zeta_{0}$. The fuel concentration equation in this reactive zone can be written in a first approximation as

$\frac{\partial^{2} y_{2}}{\partial s^{2}}=\tilde{\Delta} y_{2} \exp (-s)$,

where

$\tilde{\Delta}=\frac{L^{2} \Delta \exp \left(-\zeta_{0}{ }^{\frac{1}{L}}\right)}{\zeta_{0}{ }^{\frac{2}{L}-1}}$

and

$s=\frac{\left(\zeta-\zeta_{0}\right) \zeta_{0}^{\frac{1}{L}-1}}{L}$.

The boundary conditions transform to

$y_{2} \sim 0 \quad$ as $\sigma \rightarrow-\infty$ and

$y_{2} \sim \frac{L s}{\zeta_{0}{ }^{\frac{1}{L}-1}}-\zeta_{0}$ as $s \rightarrow \infty$.

The nondimensional radical concentration in the reactive zone is given after Eq. 42 in a first approximation as $\phi_{\infty}=\zeta_{0}$. Equations 43 and 44 represent an eigenvalue problem, the solution to which is only possible for one value of the parameter of $\tilde{\Delta}$. Equation 43 can be written as an homogeneous modified Bessel equation of order zero

$t^{2} \frac{\partial^{2} y_{2}}{\partial t^{2}}+t \frac{\partial y_{2}}{\partial t}-t^{2} y_{2}=0$

with

$t=2 \sqrt{\tilde{\Delta}} \exp \left(-\frac{s}{2}\right)$
The boundary conditions are then

$y_{2}(0) \sim \frac{L}{\zeta_{0}{ }^{\frac{1}{L}-1}}[2 \ln (2)+\ln (\tilde{\Delta})-2 \ln (t)]$,

$y_{2}(\infty) \sim 0$.

The solution of Eqs. 45 and 47 is given by $y_{2}=C K_{0}(t)$, where $K_{0}$ corresponds to the modified Bessel function of order zero, with an asymptotic behavior for small values of $t$ given as

$y_{2} \sim-C[\gamma-\ln (2)+\ln (t)]$, for $t \rightarrow 0$,

where $\gamma$ is the Euler's constant, $\gamma \simeq$ $0.57721 \ldots$. After equating the relationships 46 and 48 , we obtain the eigenvalue $\tilde{\Delta}=$ $\exp (-2 \gamma)$. Therefore,

$\Delta=\frac{\exp \left(\phi_{\infty}^{\frac{1}{L}}-2 \gamma\right) \phi_{\infty}^{\frac{2}{L}-1}}{L^{2}}$,

which gives the value of $\phi_{\infty}$, which is needed to plot the radical concentration as a function of the nondimensional coordinate.

\section{NUMERICAL SOLUTION}

In this section, Eqs. 5-9 are solved numerically using finite difference techniques. The nonlinear parabolic equations are solved iteratively. The initial profiles for $\chi=0$ are

$Y_{\mathrm{H}_{2}}=\frac{1}{2} \operatorname{erfc}\left(\frac{\sqrt{L} \eta}{2}\right)$,

$Y_{\mathrm{O}_{2}}=1-\frac{1}{2} \operatorname{erfc}\left(\frac{\eta}{2}\right)$,

$Y_{\mathrm{O}}=Y_{\mathrm{O} \infty}\left(1-\frac{1}{2} \operatorname{erfc}\left(\frac{\eta}{2}\right)\right)$.

The derivatives in the $\chi$ direction are discretized by using Euler's method. Derivatives in the $\boldsymbol{\eta}$ direction are discretized by using central differences. The right-hand side of Eqs. 5-7 are linearized by solving separately each of the equations. At the beginning of the iteration, the unknown species concentration at a 


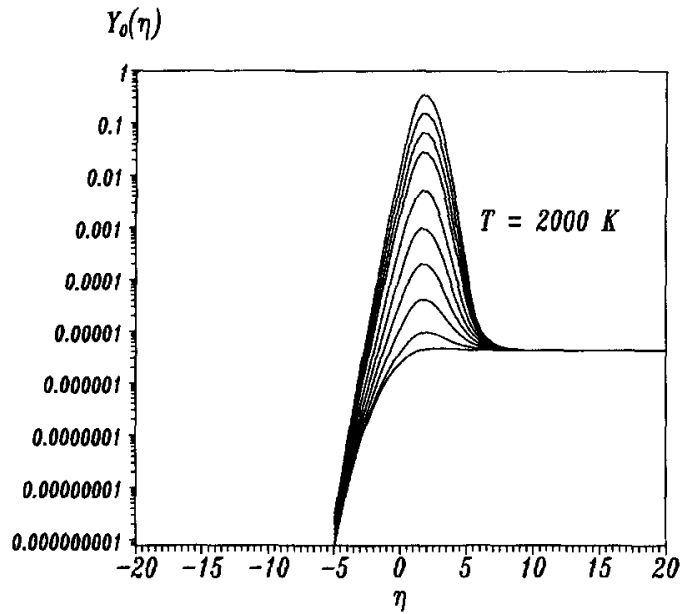

Fig. 4. Radical concentration profiles as a function of $\eta$ for different values of $\chi$.

new position $\chi$ are replaced by the known functions at a previous station, $\chi-\Delta \chi$. This procedure is repeated until convergence is achieved. The system of equations are represented by tri-diagonal matrices.

Figure 4 shows the radical concentration profiles at different values of the nondimensional coordinate, $\chi$ for $T_{\infty}=2000 \mathrm{~K}, T_{-\infty}=$ $300 \mathrm{~K}$ and a pressure of $10^{5} \mathrm{~Pa}$. The maximum radical concentration occurs at values of $\eta \sim$ 1.6. This is not a large value as assumed previously because the effective activation energy of reaction 3 is not very high being of only 71.2 $\mathrm{KJ} / \mathrm{mol}$, for $T_{\infty}=2000 \mathrm{~K}$. Figure 5 shows the computed profiles for the concentration of molecular hydrogen both at the beginning of the mixing layer and close to the ignition conditions for the same parametric set as indicated previously. The reactant consumption is very small and mainly produced in the zone of maximum radical concentration. However, the value of the molecular hydrogen concentration in the reaction zone is close to 0.3 , thus not to small to consider a very lean mixture. Because of the high diffusivity of the molecular hydrogen, reactant consumption is very small and is rapidly replaced by diffusing from the fuel side. Figure 6 shows evolution of the maximum value of the radical concentration as a function of $\chi$ for three different values of the temperature of the air stream. The big difference between them is due to the initial radical concentration at the air side due to the assumed partial

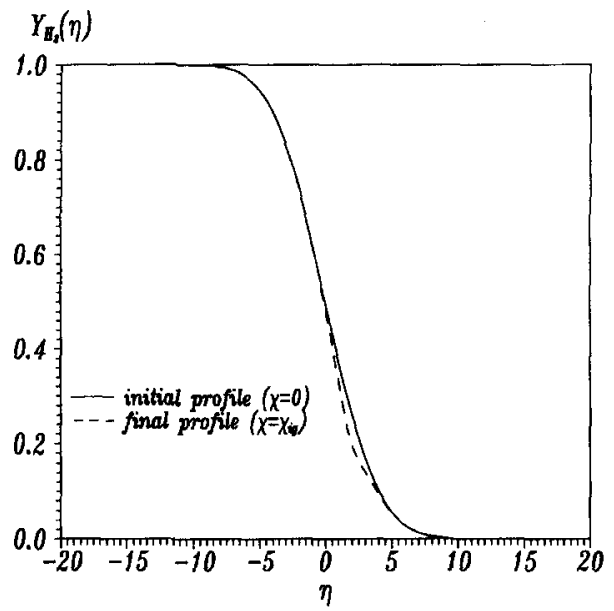

Fig. 5. Concentration profiles for molecular hydrogen at $x=0$ and $x \rightarrow \chi_{\text {ig }}$.

equilibrium between molecular and atomic oxygen. The criterion for the ignition condition used previously was related to the partial equilibrium of the shuffle reactions. However as shown in Fig. 6, the ignition condition can be defined simply when the radical concentration becomes of order unity. The error introduced in any of these criteria is small compared with the ignition distance. Figure 7 shows the nondimensional ignition distance as a function of the air temperature for two different hydrogen temperatures. The influence of the fuel temperature is not strong as long this value is not too close to the air temperature. Figure 8 shows a comparison of the evolution of the

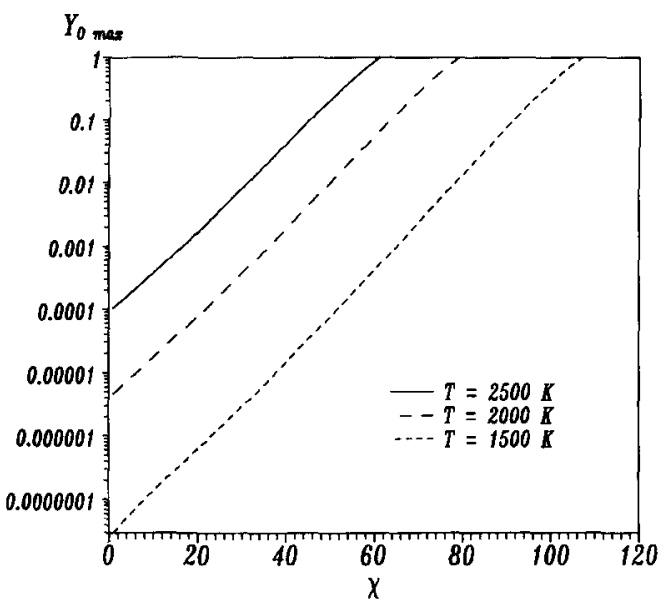

Fig. 6. Evolution of the maximum radical concentration as a function of $\chi$, for different air temperatures. 


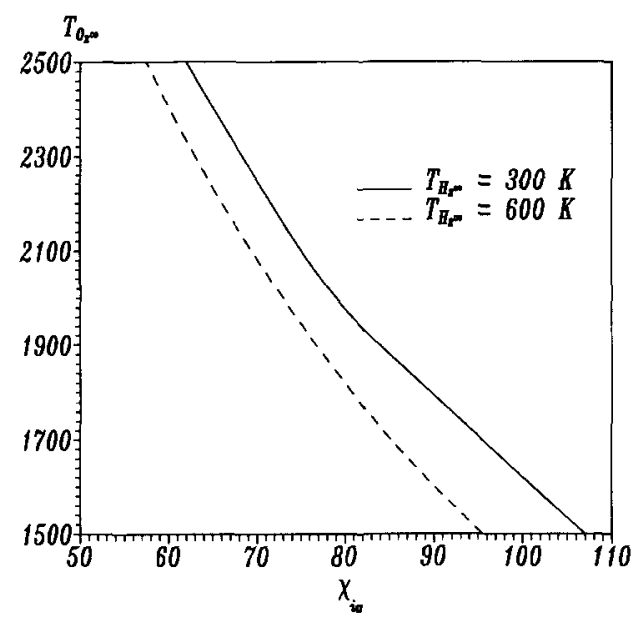

Fig. 7. Influence of reactant temperatures on the nondimensional ignition distance.

maximum radical concentration as a function of $\chi$ for the ignition process $\left(T_{\mathrm{O}_{2} \infty}=2000 \mathrm{~K}\right.$, $T_{\mathrm{H}_{2} \infty}=300 \mathrm{~K}, p=10^{5} \mathrm{~Pa}$ ) using different approximations. The solid line represents the solution with all terms included in the species conservation Equation 7. Reactant consumption as well the initiation reaction 14 and air dissociation are taken into account. Not including reactant consumption and the initiation step 14 have not a big influence on the ignition distance. In this case, air dissociation is important increasing one order of magnitude the maximum radical concentration at a given distance. However in the ignition distance, it represents an error of $10 \%$ approximately. The quasi-steady approximation (with and without

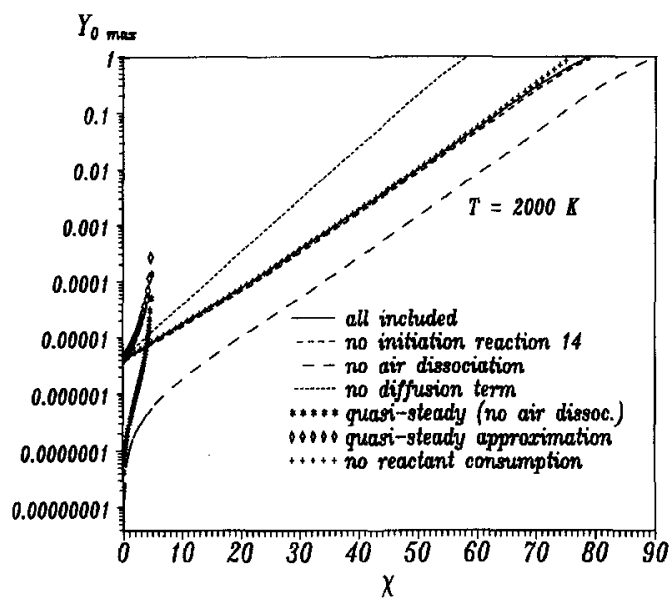

Fig. 8. Different approximations to the ignition process. air dissociation) are also plotted. The critical value, $\chi_{c} \approx 4.9$, is found to be very small compared with the ignition distance, $\chi_{c} \ll \chi_{\mathrm{ig}}$. It means that ignition is mainly a convective-reactive process. In order to see the influence of mass diffusion in the process, the solution without this term is also presented in Fig. 8. It underestimates the ignition distance by approximately $20 \%$, showing that diffusion delays slightly the ignition event in this mainly convective-reactive process.

\section{FINAL REMARKS}

In this work we study the ignition of hydrogen co-flowing with a high-temperature air in a mixing layer. Due to the relatively high sensitivity of the chemical reaction with temperature, the reaction will take place in the air side, with relatively very small concentration of molecular hydrogen. In this case the equivalence ratio is very small in regions where reaction is important and the limiting reaction switches from reaction 1 for stoichiometric and rich mixtures to reaction 3 for lean mixtures. Using a reduced kinetic mechanism valid for lean hydrogen mixtures, we obtained first a critical nondimensional distance, $\chi_{c}$, using the quasi-steady approximation. For $\chi>\chi_{c}$, diffusion plays no more any important role and the process changes from diffusive-reactive to convective-reactive. Numerical results using the evolutive equations give an ignition distance, $\chi_{\text {ig }}$, which is very large compared with $\chi_{c}$, that is $\chi_{\mathrm{ig}} \gg \chi_{c}$. This fact will allow us to develop a simplified analysis of the ignition as a pure convective-reactive process. As indicated previously, reactant consumption is not very strong due to the high diffusivity of molecular hydrogen. Assuming no reactant consumption, the convective-reactive form of the governing equation for the radical concentration, Eq. 7, takes the form

$$
\begin{aligned}
\chi \frac{\partial Y_{\mathrm{O}}}{\partial \chi} & =\chi G(\eta) \\
& =\chi Y_{\mathrm{H}_{2} f}(\eta) Y_{\mathrm{O}} \exp \left(\frac{\beta(\theta(\eta)-1)}{\theta(\eta)}\right),
\end{aligned}
$$


where the initiation step has been pulled out and only the air dissociation is considered. The solution of Eq. 53 is

$Y_{\mathrm{O}}=Y_{\mathrm{O} i}(\eta) \exp (G(\eta) \chi)$.

Here, $Y_{\mathrm{O} i}$ corresponds to the radical concentration profile at the beginning of the mixing layer, obtained from the air dissociation

$Y_{\mathrm{O} i}(\eta)=Y_{\mathrm{O} \infty}\left(1-0.5 \operatorname{erfc}\left(\frac{\eta}{2}\right)\right)$.

The maximum value of the radical concentration is obtained at the position, $\eta_{m}$ where $G\left(\eta_{m}\right)$ is maximum, $G_{m}=G\left(\eta_{m}\right)$. In order to obtain this value we make $d G / d \eta=0$, resulting the following relationship

$Y_{\mathrm{H}_{2} f}\left(\eta_{m}\right) \frac{\beta}{\theta_{m}{ }^{2}}\left(\frac{d \theta}{d \eta}\right)_{m}=-\left(\frac{d Y_{\mathrm{H}_{2} f}}{d \eta}\right)_{m}$.

Using the asymptotic approximations for the error functions, assuming large values of $\eta_{m}$, we obtain the following transcendental equation for $\eta_{m}$

$\eta_{m} \exp \left(\frac{\eta_{m}^{2}}{4}\right)=\frac{\beta \theta_{d}}{L \sqrt{\pi}}$,

which can be solved iteratively. From Eqs. 54 and 57, the nondimensional ignition distance can be approximated to

$\chi_{\mathrm{ig}} \simeq-\frac{\ln \left(Y_{\mathrm{o}}\left(1-\frac{L}{\beta \theta_{d}}\right)\right) \exp \left(\frac{\eta_{m}{ }^{2}}{4}(1+L)\right)}{\sqrt{\pi L} \eta_{m}}$.

In physical units, the ignition distance $x_{\text {ig }}$ is given finally by

$U_{\infty} W_{\mathrm{H}_{2}} R T_{\infty} \exp \left(\frac{\bar{E}_{3}}{R T_{\infty}}+\frac{\eta_{m}{ }^{2}}{4}(1+L)\right)$
$\ln \left(Y_{\mathrm{o} \infty}\left(1-\frac{L}{\beta \theta_{d}}\right)\right)$

where $p$ is the pressure and $\bar{W}$ is the molecular weight of the mixture, $\bar{W} \approx 29$.
The ignition distance increases linearly as pressure decreases and dilution in the fuel side increases. Figure 9 shows the ignition distance calculated with Eq. 59 compared with that obtained numerically, for different values of the air and fuel temperatures and for $U_{\infty}=500$ $\mathrm{m} \mathrm{s}^{-1}, p=1$ bar and $y_{\mathrm{H}_{2 \infty}}=1$. This figure shows the right trends in the parametric space and very good comparison between the analytical and numerical solutions. The critical distance using the quasi-steady approximation is also plotted. The ignition distance given by Eq. 59 has been obtained for the asymptotic limit of high values of the Zeldovich number $\beta$. However, the effective activation energy is only $71.2 \mathrm{KJ} / \mathrm{mol}$, for $T_{\infty}=2000 \mathrm{~K}$. This gives a Zeldovich number of $\beta \approx 4.28$. In most of the calculations, the computed value of $\eta_{m}$ is of order unity and not much larger than unity as the asymptotic model requires. This could introduce errors in the quantification of the ignition distance, but the trends are correct and Eq. 59 represents the first attempt to obtain a closed form solution for the high-temperature ignition of hydrogen in mixing layers. Another source of error could be that reaction 1 is not very strong to support the quasi-steady behavior of the atomic hydrogen. In this case, Eq. 59 would underestimate the ignition distance due to the finite rate of reaction 1 . We did in this work the simplest iso-velocity case, because the solution is not very sensitive to variations in velocity due to the large temperature difference between both reactants. Because the

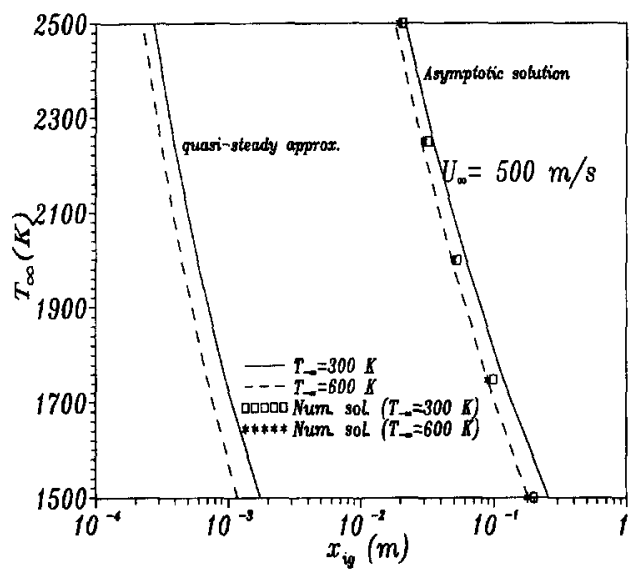

Fig. 9. Ignition distance using analytical (Eq. 59) and numerical results. 
Lewis number of the molecular hydrogen is very low compared with unity, the thickness of the fuel mixing layer is much wider than that of the viscous layer. The chemical reaction is very sensitive to the temperature and thus the reaction zone is pushed to the air side where the fuel concentration is still high enough and the mixture velocity correponds to the air velocity. This is the reason why the process could be analyzed using a reaction-convection balance, while diffusion plays only a secondary role. The characteristic velocity of the process to be used in Eq. 59 is then the air velocity.

We thank to E. Luna for his help in the numerical calculations. This work was supported by CONACYT, Mexico under Grant 0904-A9110.

\section{REFERENCES}

1. Hussaini, M. Y., Kumar, A., Voigt, R. G., Eds., Major Research Topics in Combustion, Springer Verlag, 1992.

2. Baulch, D. L., Drysdale, D. D., Horne, D. G., and Lloyd, A. C., Evaluated Kinetic Data for High Temperature Reactions, Vol. 1: Homogeneous Gas Phase Reactions of the $H-O$ System, Butherworths, London, 1972.

3. Warnatz, J., in Combustion Chemistry (W. C. Gardiner, Ed.), Springer, New York, 1984, pp. 197-360.

4. Dixon-Lewis, G., and Williams, D. J., in Comprehensive Chemical Kinetics, Vol. 17: The Oxidation of Hydrogen and Carbon Monoxide (C. H. Bamford and C. F. H. Tipper, Eds.), Elsevier, Oxford.

5. Treviño, C., and Williams, F. A., Prog. Astronaut. Aeronaut. 113:129-165 (1988).

6. Seshadri, K., and Peters, N., Combust. Flame 73:23-44 (1988).
7. Brokaw, R. S., Tenth (Symposium) International on Combustion, The Combustion Institute, 1965, pp. 269-278.

8. Gardiner, W. C., Wakefield, C. B., and Walker, B. F., in Shock Waves in Chemistry and Chemical Technology (A. Lifshitz, Ed.), Marcel Dekker, New York, 1981.

9. Kailasaneth, K., Oran, E. S., Boris, J. P., and Young, T. R., in Numerical Methods in Laminar Flame Propagation (N. Peters and J. Warnatz, Eds.), Vieweg, Braunschweig, p. 152.

10. Wiriyawit, S., and Dabora, E. K., Twentieth Symposium (International) on Combustion, The Combustion Institute, Pittsburgh, 1984, pp. 179-186.

11. Maas, U., and Warnatz, J., Combust. Flame 74:53 (1988).

12. Peters, N., Lecture Notes Phys., Springer, New York, 241:90-109 (1985).

13. Peters, N., and Williams, F. A., Combust. Flame Vol. 68:185-207 (1987).

14. Treviño, C., Prog. Astronaut. Aeronaut. 131:19-43 (1991).

15. Berman, V. S., and Ryazanstsev, Yu. S., Fluid Dyn. 12:758 (1978).

16. Law, C. K., and Law, H. K., J. Fluid Mech. 92:97 (1979).

17. Méndez, F., Treviño, C., and Liñán, A., Combust. Sci. Technol. 48:129-149 (1986).

18. Zeldovich, Y. B., "Teorii Rasprostranenia Plameni," Zhurnal Fizicheskoi Khimii (USSR), T 22:27. Published also as NACA Tech. Memo (1951), p. 1282.

19. Niioka, T., Combust. Flame 76:143-149 (1988).

20. Treviño, C., and Méndez, F., Combust. Sci. Technol. 78:197-216 (1991).

21. Treviño, C., and Liñán, A., in Combustion in HighSpeed Flows (J. Buckmaster, T. L. Jackson, and A. Kumar, Eds.), Kluwer, Dordrecht, The Netherlands, 1994, pp. 477-490. 